\title{
Anti-tumour necrosis factor activity in saliva of various tick species and its appearance during the feeding period
}

\author{
Markéta Rezková and Jan Kopecký \\ Faculty of Science, University of South Bohemia, České Budějovice, Czech Republic
}

\begin{abstract}
Tumour necrosis factor (TNF) plays a central role in the inflammatory process. In the skin, it contributes to immune containment of tick-transmitted pathogens like Borrelia burgdorferi. In the saliva of some tick species, active compounds are present that inhibit detection of TNF in specific ELISA. We compared the presence of anti-TNF activity in saliva or salivary gland extract from 11 tick species from the family Ixodidae and demonstrated it in genera Ixodes Latreille, 1795 and Haemaphysalis Koch, 1844. Analysis of anti-TNF activity in Ixodes ricinus (Linnaeus, 1758) saliva during the feeding period showed that it is present in the late, rapid phase of engorgement. Significance of anti-TNF activity for tick feeding and transmission of tick-borne pathogens is discussed.
\end{abstract}

Keywords: ixodid ticks, cytokine, immunomodulation

Ticks transmit a broad variety of infectious agents of medical and veterinary importance. In Europe, the most important pathogens transmitted by ticks are Borrelia burgdorferi sensu lato, tick-borne encephalitis virus, Anaplasma phagocytophilum and Babesia spp. (Michelet et al. 2014). Tick saliva contains numerous pharmacologically active molecules able to modulate the host defences of pain and itch, haemostasis, inflammation, innate and adaptive immunity and wound healing (Steen et al. 2006, Wikel 2013). First interactions with the host immune system occur at the cutaneous interface in the complex sequence of host defence responses and tick countermeasures resulting in an environment that facilitates successful blood feeding and pathogen transmission.

The immunomodulatory molecules in tick saliva affect both innate and adaptive immunity of the host. Ticks counteract cutaneous and systemic immune defences involving keratinocytes, neutrophils, mast cells, basophils, natural killer cells, dendritic cells, macrophages, endothelial cells, T and B lymphocytes, cytokines, chemokines and complement (Francischetti et al. 2009, Wikel 2013).

Anti-inflammatory effect of tick saliva very likely contributes to the promotion of tick-borne pathogen transmission (Kern et al. 2011). Among the factors possibly responsible for the anti-inflammatory effect of tick saliva, histamine-binding proteins (Paesen et al. 1999), complement-inhibitory factors (Ribeiro 1987), molecules inhibiting production of alarmins and various cytokines produced by keratinocytes (Marchal et al. 2011, Bernard et al. 2016) or cytokine-binding proteins (Hajnická et al. 2005, Déruaz et al. 2008) can be considered.
Tumor necrosis factor (TNF) plays a central role in inflammatory processes. Many of the proinflammatory effects of TNF can be explained on the basis of its influence on vascular endothelium-leukocyte interactions. In response to TNF, endothelial cells display various adhesion molecules including E-selectin, ICAM-1 and VCAM-1. This, in combination with the release of chemokines, leads to the recruitment of different populations of leukocytes independent of antigen recognition (Bradley 2008).

TNF plays an important role in the protection against tick-transmitted pathogens. TNF injected into mice infected with $B$. burgdorferi via ticks reduced transmission of spirochetes by $95 \%$ (Zeidner et al. 1996). In the saliva of the Ixodes ricinus (Linnaeus, 1758) tick anti-TNF activity was demonstrated in both ELISA and bioassay (Koník et al. 2006). It is very likely that anti-TNF activity in tick saliva can influence recruitment of inflammatory cells into the tick feeding site (Severinová et al. 2005, Vachiery et al. 2015). During evolution, several strategies have developed in different organisms to counter TNF activities. They operate by inhibiting TNF ligand and its receptors or by modulating TNF-activated signaling pathways (Rahman et al. 2006). For example poxviruses developed TNF binding proteins blocking interaction of the cytokine with its receptor (Rahman et al. 2006). In humans, TNF-binding protein was found in plasma and urine (Peetre et al. 1988).

In this paper we present the comparison of anti-TNF activity in saliva/ salivary gland extract (SGE) of 11 tick species belonging to the family Ixodidae and dynamics of the presence of TNF inhibitory activity in SGE from the tick Ixodes ricinus.

Address for correspondence: J. Kopecký, Faculty of Science, University of South Bohemia, Branišovská 1760, 37005 České Budějovice, Czech Republic. Phone: +420387776274; E-mail: jkopec@jcu.cz 

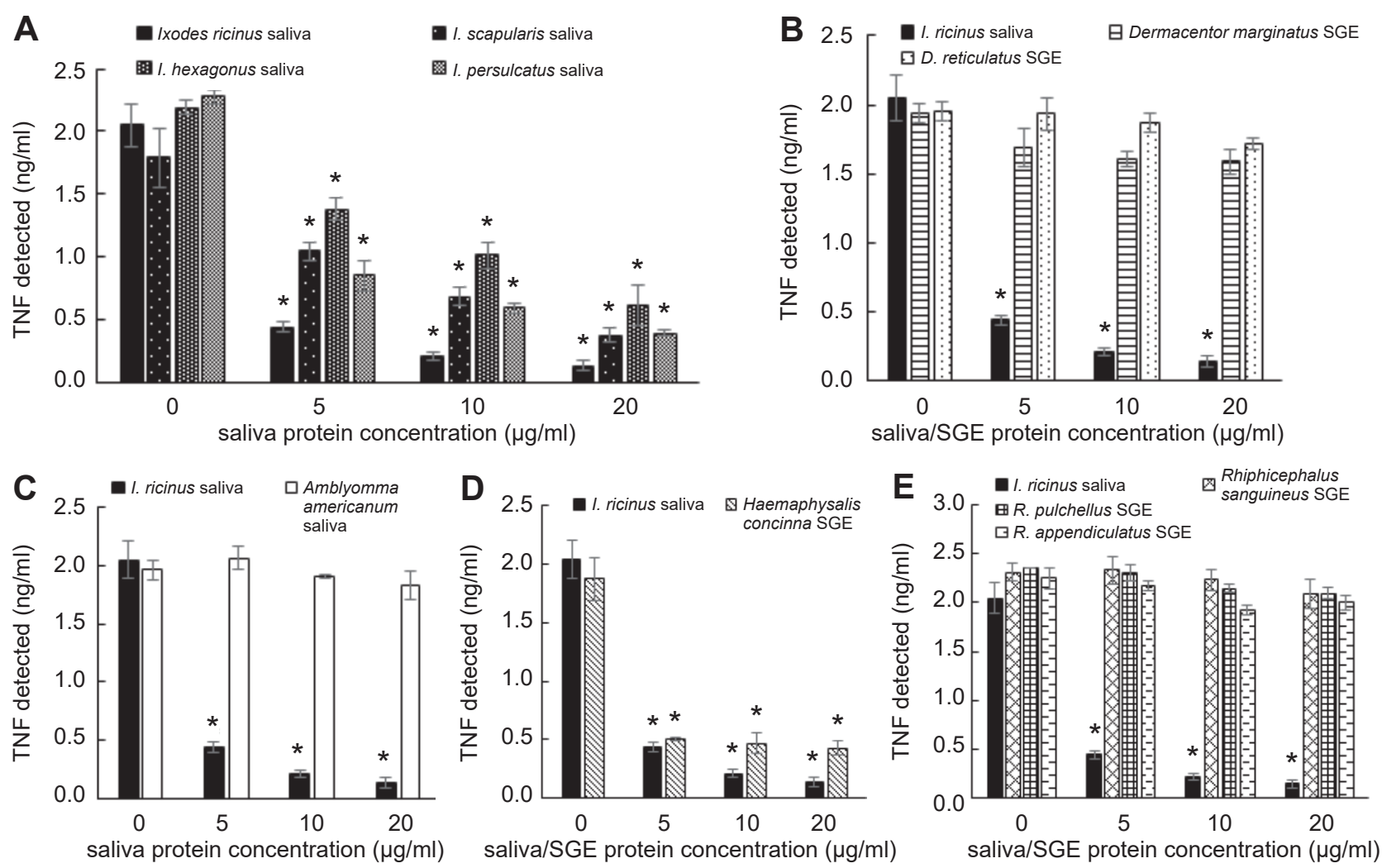

Fig. 1. Anti-tumour necrosis factor (TNF) activity in saliva/salivary gland extract (SGE) from various tick species as determined by an ELISA. Ticks (females) were fed on guinea pigs to partial engorgement (6-7 days). Various concentrations of saliva/SGE protein were preincubated for $1.5 \mathrm{~h}$ with $2 \mathrm{ng} / \mathrm{ml}$ of mouse recombinant TNF before the cytokine ELISA was performed. Saliva of Ixodes ricinus (Linnaeus, 1758) was used as a positive control. PBS instead of saliva/SGE was added into negative control. Results are presented as the mean \pm SD of triplicate wells. *The difference between saliva-treated and untreated group was significant at $\mathrm{P}<0.05$.

\section{MATERIALS AND METHODS}

\section{Tick saliva and salivary gland extract}

The following tick species from the colony of the Institute of Parasitology, Biology Centre, Czech Academy of Sciences in České Budějovice: Amblyomma americanum (Linnaeus, 1758), Dermacentor marginatus (Sulzer, 1776), D. reticulatus (Fabricius, 1794), Haemaphysalis concinna Koch, 1844, I. ricinus, I. persulcatus (Schulze, 1930), I. hexagonus (Leach, 1815), I. scapularis (Say, 1821), Rhipicephalus appendiculatus Neumann, 1901, R. pulchellus (Gerstäcker, 1873) and R. sanguineus (Latreille, 1806) were used in the present study. Ticks were fed in groups of mating pairs within retaining cells attached to the backs of guinea pigs. Partially fed (6-7 days) females were removed and either salivation was induced or salivary gland extract (SGE) was prepared.

For preparation of saliva, ticks were immobilised, $10 \mathrm{ml}$ capillary tubes (Sigma-Aldrich Co., St. Louis, Missouri, USA) was fitted over their mouthparts, and $2 \mathrm{ml}$ of $5 \%$ pilocarpine was applied topically to their dorsa. Saliva was collected over $2 \mathrm{~h}$ in a $37^{\circ} \mathrm{C}$ environment, pooled and stored at $-70^{\circ} \mathrm{C}$ until used. As pilocarpine was present in the saliva in about $80 \mathrm{mM}$ concentration, its effect on the detection of TNF by ELISA was tested. No influence on the results was observed.

For SGE preparation, ticks were washed in $15 \%$ ethanol and salivary glands were dissected from ticks and pooled. After washing in phosphate buffered saline (PBS) the salivary glands were homogenised in $500 \mathrm{ml}$ of PBS by sonication in Ultrasonic Pro- cessor UP200S (Hielscher Ultrasonics, Teltow, Germany) and clarified by centrifugation at $10,000 \times g$ for $10 \mathrm{~min}$. The protein concentration of clarified SGE or saliva was determined using a protein estimation kit with the Bradford reagent (Bio-Rad, Richmond, USA). Neither a protease inhibitor cocktail for use in tissue culture media containing aprotinin, bestatin, leupeptin, E-64, and pepstain A (P-1860, Sigma-Aldrich) added to the saliva or SGE at the final dilution 100 -fold nor the inhibitor of metalloproteases EDTA in the final concentration $10 \mathrm{mM}$ influenced the anti-TNF activity in saliva or SGE.

\section{Cytokine ELISA}

Antibody-sandwich ELISA using commercial ELISA Kit Ready-Set-Go (eBioscience, San Diego, USA) was used for TNF measurement. To estimate the TNF-inhibitory effect of tick saliva or SGE, concentrations of 5,10 and $20 \mathrm{mg} / \mathrm{ml}$ PBS were preincubated with $2 \mathrm{ng} / \mathrm{ml}$ TNF (recombinant mouse tumor necrosis factor-a, Gibco, Langley, Oklahoma, USA) for $1.5 \mathrm{~h}$ at room temperature. After incubation, $50 \mathrm{ml}$ aliquots were applied to the ELISA plate in triplicate. ELISA was performed according to manufacturer's instructions.

\section{Statistical analysis}

In all experiments samples were tested in triplicates. The significance obtained between experimental groups was evaluated by analysis of variance (one-way ANOVA) and Tukey post hoc test using Statistica 8.0 software (StatSoft, Tulsa, OK, USA). The results were considered significant when $\mathrm{P}<0.05$. 


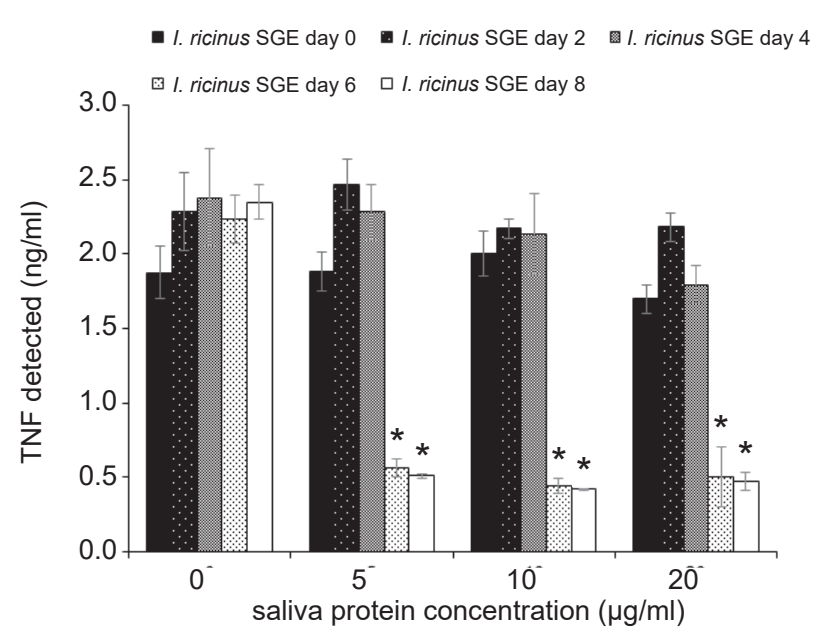

Fig. 2. Anti-tumour necrosis factor (TNF) activity in tick salivary glands during feeding. Salivary gland extract (SGE) was prepared from females of Ixodes ricinus (Linnaeus, 1758) either unfed (day 0) or fed for 2, 4, 6 and 8 days. Various concentrations of SGE protein were preincubated for $1.5 \mathrm{~h}$ with $2 \mathrm{ng} / \mathrm{ml}$ of mouse recombinant TNF before the cytokine ELISA was performed. PBS instead of SGE was added into negative control. Results are presented as the mean $\pm \mathrm{SD}$ of triplicate wells. *The difference between saliva-treated and untreated group was significant at $\mathrm{P}<0.05$.

\section{RESULTS}

\section{Anti-TNF activity in various tick species}

Comparison of the effect of saliva from four representatives of genus Ixodes Latreille, 1795 showed that the weakest anti-TNF activity is present in saliva from $I$. hexagonus followed by I. scapularis and I. persulcatus. The strongest TNF-inhibitory activity was found in I. ricinus saliva (Fig. 1A), which was further used as a positive control. Examining the anti-TNF activity in SGE from additional ixodid tick species, Dermacentor marginatus and D. reticulatus, showed no activity in all SGE concentrations tested (Fig. 1B). Similar results were obtained with saliva from the lone star tick Amblyomma americanum (Fig. 1C).

By contrast, a strong TNF-inhibitory activity was demonstrated in SGE from Haemaphysalis concinna, the known vector of Francisella tularensis and Rickettsia sibirica. It reduced measurable TNF activity by more than 75\% (Fig. 1D)

The last ixodid tick species tested were those of the genus Rhipicephalus Koch, 1844. None of three species ( $R$. sanguineus, $R$. appendiculatus, $R$. pulchellus) exhibited anti-TNF activity (Fig. 1E).

The results obtained in all 11 tick species are summarised in Table 1. It can be concluded that anti-TNF could be detected only in species of the genera Ixodes and Haemaphysalis Koch, 1844, whereas genera Amblyomma Koch, 1844, Dermacentor Koch, 1844, and Rhipicephalus lack the capacity to reduce measurable activity of TNF in ELISA.
Table 1. Anti-tumour necrosis factor (anti-TNF) activity in sali$\mathrm{va} /$ salivary gland extract (SGE) of various tick species.

\begin{tabular}{lc}
\hline Tick species & Anti-TNF activity \\
\hline Amblyomma americanum (Linnaeus, 1758) & - \\
Dermacentor marginatus (Sulzer, 1776) & - \\
Dermacentor reticulatus (Fabricius, 1794) & - \\
Haemaphysalis concinna Koch, 1844 & + \\
Ixodes hexagonus (Leach, 1815) & + \\
Ixodes persulcatus (Schulze, 1930) & + \\
Ixodes ricinus (Linnaeus, 1758) & + \\
Ixodes scapularis (Say, 1821) & + \\
Rhipicephalus appendiculatus Neumann, 1901 & - \\
Rhipicephalus pulchellus (Gerstäcker, 1873) & - \\
Rhipicephalus sanguineus (Latreille, 1806) & - \\
\hline
\end{tabular}

Saliva or SGE from partially fed (6-7 days) females was tested for anti-TNF activity by TNF ELISA. Results are expressed as the presence $(+)$ or absence (-) of anti-TNF activity.

\section{Dynamics of anti-TNF activity in tick salivary glands during tick feeding}

SGE was prepared from I. ricinus ticks feeding for 2, 4,6 and 8 days and from unfed ticks. Anti-TNF activity determined by ELISA is shown in Fig. 2. TNF-inhibitory activity was present in SGE from day 6 to the end of the feeding period (day 8), i.e. in the late, rapid feeding phase.

\section{DISCUSSION}

Anti-TNF activity discovered by Koník et al. (2006) represents one of numerous anti-inflammatory and immunomodulatory mechanisms that ticks developed to facilitate feeding on blood. TNF plays an irreplaceable role in inflammation that is also integral part of cutaneous basophil hypersensitivity, a specific form of delayed type of hypersensitivity which can lead to rejection of the feeding tick (Ferreira et al 2003). TNF exerts its effect on tick-transmitted pathogens indirectly, through the induction, recruitment and activation of inflammatory cells (Hehlgans and Pfeffer 2005).

In saliva of various tick species, several cytokine-binding molecules have been discovered. For example IL-2 binding protein was found in saliva of I. scapularis (see Gillespie et al. 2001), chemokine-binding molecules termed evasins were identified in saliva of Rhipicephalus sanguineus (see Déruaz et al. 2008), in eight tick species of Rhipicephalus and Amblyomma (see Singh et al. 2017) and other ixodid ticks (Vančová et al. 2010). Some of these anti-inflammatory molecules possess a strong therapeutic potential (Bonvin et al. 2016)

The knowledge about existence of anti-TNF activity was extended from I. ricinus to other ixodid tick species, Haemaphysalis concinna, I. hexagonus, I. persulcatus and I. scapularis. It is of interest that anti-TNF activity correlates with the ability of particular tick species to serve as a competent vector of Borrelia burgdorferi (see Eisen and Lane 2002). The only exception is Haemaphysalis concin$n a$ in which spirochete transmission has not been demonstrated so far (Sun and Xu 2003). 
Monitoring of anti-TNF activity during feeding of I. ricinus revealed interesting data. Although TNF belongs to early cytokines released from macrophages within few hours after stimulation (Estrada et al. 1998), anti-TNF activity appeared in SGE in the late, fast feeding phase from day 6 after feeding commencement. The reason for this late appearance of anti-TNF activity can be the procoagulant activity of the cytokine. TNF induces expression of tissue factor, the initiator of blood coagulation cascade while suppressing endothelial cell-dependent protein $\mathrm{C}$ activation and anticoagulant function on the cell surface. It leads to promotion of clot formation (Nawroth and Stern 1986). Administration of anti-TNF activity via saliva into the wound can facilitate blood feeding. TNF also controls migration of Langerhans cells from the skin to draining lymph nodes (Cumberbatch and Kimber 1995), where they initiate adaptive immunity to tick saliva antigens.

It should be considered that ticks possess other yet unknown molecule(s) in the saliva able to block/downregulate expression of TNF in very early phase of tick feeding. Very strong suppression (96\%) of TNF production by murine peritoneal macrophages stimulated with lipopolysaccharide (LPS) was recorded using SGE from unfed Dermacentor andersoni (see Ramachandra and Wikel 1992). Even though saliva inhibitory effect on TNF production was usually shown by an ELISA, it cannot be explained by TNF-binding activity, because it was also demonstrated on the cytokine mRNA level (Fuschsberger et al. 1995). Gwakisa et al. (2001) showed anti-TNF activity of SGE of Rhipicephalus appendiculatus on both protein and cytokine mRNA level. In I. ricinus the anti-TNF activity was displayed by a serpin which bound to monocytes/macrophages and inhibited their ability to secrete TNF (Prevot et al. 2009). It seems likely that anti-TNF activity in SGE of species of Dermacentor and Rhipicephalus is not connected with TNF-blocking activity demonstrated by us in other tick species, but with the inhibitory effect on TNF expression.

Even though the inhibition of TNF activity through binding of the tick salivary compound(s) to TNF is connected with the late phase of tick feeding, it can still support the growth of spirochetes in the host skin and their spreading throughout the body. Spirochetes remain localised in the skin for several days to weeks before dissemination to target organs (Steere et al. 2004). Interestingly, the presence of tick saliva in the skin delays and decreases the peak of Borrelia multiplication of borreliae in comparison with syringe inoculated spirochetes (Kern et al. 2011). Spirochetes transmitted via infected nymphs of I. ricinus were detectable in mouse skin from day 7 to day 30 after feeding commencement. From these data it can be concluded that anti-TNF activity in tick saliva could influence growth and spreading of spirochetes due to its anti-inflammatory effect.

Identification of anti-TNF molecule in tick saliva remains to be done. A protein binding TNF and preventing binding of detection monoclonal antibody can be responsible for this activity (Koník et al. 2006). This protein should be also able to prevent binding of TNF to its receptor on target cell thus inhibiting TNF bioassay. On the other hand, TNF, which is bioactive as a trimer, can be cleaved by a protease what leads to the loss of biological activity and the epitope recognised by detection antibody. As we were not able to affect salivary anti-TNF activity by treatment with either protease inhibitor cocktail or metalloprotease inhibitor EDTA, we presume the anti-TNF activity detected by ELISA was due to a TNF-binding protein.

Acknowledgements. We are greatly indebted to Jan Erhart for providing ticks from the colony kept in the Institute of Parasitology, Biology Centre of the Czech Academy of Sciences, České Budějovice.

\section{REFERENCES}

Bernard Q., Gallo R.L., Jaulhac B., Nakatsuji T., Luft B., YANG X., Boulanger N. 2016: Ixodes tick saliva suppresses the keratinocyte cytokine response to TLR2/TLR3 ligands during early exposure to Lyme borreliosis. Exp. Dermatol. 25: 26-31.

Bonvin P., Power C.A., Proudfoot A.E. 2016: Evasins: therapeutic potential of a new family of chemokine-binding proteins from ticks. Front. Immunol. 7: 208.

Bradley J.R. 2008: TNF-mediated inflammatory disease. J. Pathol. 214: 149-160.

Cumberbatch M., Kimber I. 1995: Tumour necrosis factor-alpha is required for accumulation of dendritic cells in draining lymph nodes and for optimal contact sensitization. Immunology 84: 31-35.

Déruaz M., Frauenschuh A., Alessandri A.L., Dias J.M., Coelho F.M., Russo R.C., Ferreira B.R., Graham G.J., Shaw J.P., Wells T.N., Teixeira M.M., Power C.A., ProudFOOT A.E. 2008: Ticks produce highly selective chemokine binding proteins with antiinflammatory activity. J. Exp. Med. 205: 2019-2031.

EISEN L., LANE R.S. 2002: Vectors of Borrelia burgdorferi sensu lato. In: J. Gray, O. Kahl, R.S. Lane and G. Stanek (Eds.), Lyme
Borreliosis: Biology, Epidemiology and Control. CABI Publishing, Wallingford, pp. 92-99.

Estrada A., Van Kessel A., Yun C.M., Li B. 1998: Effect of endotoxin on cytokine production and cell dynamics in mice. Immunopharmacol. Immunotoxicol. 20: 217-231.

Ferreira B.R., Szabó M.J., Cavassani K.A., Bechara G.H., Silva J.S. 2003: Antigens from Rhipicephalus sanguineus ticks elicit potent cell-mediated immune responses in resistant but not in susceptible animals. Vet. Parasitol. 115: 35-48.

Francischetti I.M., Sa-Nunes A., Mans B.J., Santos I.M., Ribeiro J.M. 2009: The role of saliva in tick feeding. Front. Biosci. 14: 2051-2088.

Fuchsberger N., Kita M., Hajnická V., Imanishi J., LabuDA M., NutTall P.A. 1995: Ixodid tick salivary gland extracts inhibit production of lipopolysaccharide-induced mRNA of several different human cytokines. Exp. Appl. Acarol. 19: 671-676.

Gillespie R.D., Dolan M.C., Piesman J., Titus R.G. 2001: Identification of an IL-2 binding protein in the saliva of the Lyme disease vector tick, Ixodes scapularis. J. Immunol. 166: 4319-4326.

Gwakisa P., Yoshihara K., Long To T., Gotoh H., Amano F., Момотаni E. 2001: Salivary gland extract of Rhipicephalus appendiculatus ticks inhibits in vitro transcription and secretion 
of cytokines and production of nitric oxide by LPS-stimulated JA-4 cells. Vet. Parasitol. 99: 53-61.

Hajnická V., Vančová I., Kocáková P., Slovák M., Gasperík J., Sláviková M., Hails R.S., Labuda M., Nuttall P.A. 2005: Manipulation of host cytokine network by ticks: a potential gateway for pathogen transmission. Parasitology 130: 333-342.

Hehlgans T., Pfeffer K. 2005: The intriguing biology of the tumour necrosis factor/tumour necrosis factor receptor superfamily: players, rules and the games. Immunology 115: 1-20.

Kern A., Collin E., Barthel C., Michel C., Jaulhac B., Boulanger N. 2011: Tick saliva represses innate immunity and cutaneous inflammation in a murine model of Lyme disease. Vector Borne Zoonotic Dis. 11: 1343-1350.

Koník P., Slavíková V., SAlát J., ŘezníčKová J., DvorožŇáKovÁ E., KopeckÝ J. 2006: Anti-tumour necrosis factor-alpha activity in Ixodes ricinus saliva. Parasite Immunol. 28: 649-656.

Marchal C., Schramm F., Kern A., Luft B.J., Yang X., Schuijt T.J., Hovius J.W., Jaulhac B., Boulanger N. 2011: Antialarmin effect of tick saliva during the transmission of Lyme disease. Infect. Immun. 79: 774-785.

Michelet L., Delannoy S., Devillers E., Umhang G., Aspan A., Juremalm M., Chirico J., van der Wal F.J., Sprong H., Boye Pihl T.P., Klitgaard K., Bødker R., Fach P., Moutailler S. 2014: High-throughput screening of tick-borne pathogens in Europe. Front. Cell. Infect. Microbiol. 4: 103.

Nawroth P.P., Stern D.M. 1986: Modulation of endothelial cell hemostatic properties by tumor necrosis factor. J. Exp. Med. 163 : $740-745$.

Paesen G.C., Adams P.L., Harlos K., Nuttall P.A., Stuart D.I. 1999: Tick histamine-binding proteins: isolation, cloning, and three-dimensional structure. Mol. Cell 3: 661-671.

Peetre C., Thysell H., Grubb A., Olsson I. 1988: A tumor necrosis factor binding protein is present in human biological fluids. Eur. J. Haematol. 41: 414-419.

Prevot P.P., Beschin A., Lins L., Beaufays J., Grosjean A., Bruys L., Adam B., Brossard M., Brasseur R., Zouaoui Boudjeltia K., Vanhamme L., Godfroid E. 2009: Exosites mediate the anti-inflammatory effects of a multifunctional serpin from the saliva of the tick Ixodes ricinus. FEBS J. 276: 3235-3246.

Rahman M.M., McFadden G. 2006: Modulation of tumor necrosis factor by microbial pathogens. PLoS Pathog. 2: e4.
RAMACHANDRA R.N., Wikel S.K. 1992: Modulation of host-immune responses by ticks (Acari: Ixodidae): effect of salivary gland extracts on host macrophages and lymphocyte cytokine production. J. Med. Entomol. 29: 818-826.

Ribeiro J.M. 1987: Ixodes dammini: salivary anti-complement activity. Exp. Parasitol. 64: 347-353.

Severinová J., Salát J., Kročová Z., Řezníč́ová J., Demová H., Horká H., KopeckÝ J. 2005: Co-inoculation of Borrelia afzelii with tick salivary gland extract influences distribution of immunocompetent cells in the skin and lymph nodes of mice. Folia Microbiol. 50: 457-463.

Singh K., Davies G., Alenazi Y., Eaton J.R.O., KawamuRA A., Bhattacharya S. 2017: Yeast surface display identifies a family of evasins from ticks with novel polyvalent CC chemokine-binding activities. Sci. Rep. 7: 4267.

Steen N.A., Barker S.C., Alewood P.F. 2006: Proteins in the saliva of the Ixodida (ticks): pharmacological features and biological significance. Toxicon 47: 1-20.

Steere A.C., Coburn J., Glickstein L. 2004: The emergence of Lyme disease. J. Clin. Invest. 113: 1093-1101.

Sun Y., Xu R. 2003: Ability of Ixodes persulcatus, Haemaphysalis concinna and Dermacentor silvarum ticks to acquire and transstadially transmit Borrelia garinii. Exp. Appl. Acarol. 31: 151-160.

Vachiery N., Puech C., Cavelier P., Rodrigues V., Aprelon R., Lefrançois T., Martinez D., Epardaud M. 2015: An in vitro model to assess the immunosuppressive effect of tick saliva on the mobilization of inflammatory monocyte-derived cells. Vet. Res. 46: 117.

Vančová I., Hajnická V. Slovák M., Nuttall P.A. 2010: Anti-chemokine activities of ixodid ticks depend on tick species, developmental stage, and duration of feeding. Vet. Parasitol. 167: 274-278.

WikeL S. 2013: Ticks and tick-borne pathogens at the cutaneous interface: host defences, tick countermeasures, and a suitable environment for pathogen establishment. Front. Microbiol. 4: 337.

Zeidner N., Dreitz M., Belasco D., Fish D. 1996: Suppression of acute Ixodes scapularis-induced Borrelia burgdorferi infection using tumor necrosis factor-alpha, interleukin-2, and interferon-gamma. J. Infect. Dis. 173: 187-195.

Cite this article as: Rezková M., Kopecký J. 2017: Anti-tumour necrosis factor activity in saliva of various tick species and its appearance during the feeding period. Folia Parasitol. 64: 032. 